Article

\title{
Effects of Salicylic Acid and Methyl Jasmonate Treatments on Flavonoid and Carotenoid Accumulation in the Juice Sacs of Satsuma Mandarin In Vitro
}

\author{
Risa Yamamoto ${ }^{1,+}$, Gang Ma ${ }^{1,2,{ }^{\dagger}}$, Lancui Zhang ${ }^{2}$, Miki Hirai ${ }^{2, \ddagger}$, Masaki Yahata ${ }^{1,2}$, \\ Kazuki Yamawaki ${ }^{1,2}$, Takehiko Shimada ${ }^{3}$, Hiroshi Fujii ${ }^{3}$, Tomoko Endo ${ }^{3}$ and Masaya Kato ${ }^{1,2, *}$ \\ 1 Graduate School of Integrated Science and Technology, Department of Bioresource Sciences, \\ Faculty of Agriculture, Shizuoka University, 836 Ohya, Suruga, Shizuoka 422-8529, Japan; \\ Yamamoto.risa.15@shizuoka.ac.jp (R.Y.); ma.gang@shizuoka.ac.jp (G.M.); \\ yahata.masaki@shizuoka.ac.jp (M.Y.); yamawaki.kazuki@shizuoka.ac.jp (K.Y.) \\ 2 Department of Bioresource Sciences, Faculty of Agriculture, Shizuoka University, 836 Ohya, Suruga, \\ Shizuoka 422-8529, Japan; zhang.lan.cui@shizuoka.ac.jp (L.Z.); miki1_hirai@pref.shizuoka.lg.jp (M.H.) \\ 3 National Institute of Fruit Tree Science (NIFTS), \\ National Agriculture and Bio-Oriented Research Organization (NARO), Shizuoka, \\ Shizuoka 424-0292, Japan; tshimada@affrc.go.jp (T.S.); hfujii@affrc.go.jp (H.F.); tomoen@affrc.go.jp (T.E.) \\ * Correspondence: kato.masaya@shizuoka.ac.jp; Tel.: +81-54-238-4830 \\ + These authors contributed equally: Risa Yamamoto and Gang Ma. \\ $\ddagger$ Current address: Shizuoka Prefectural Agriculture and Forestry Research Institute, \\ Fruit Tree Research Center, Mobata, Shimizu-ku, Shizuoka 424-0101, Japan.
}

Received: 30 October 2020; Accepted: 10 December 2020; Published: 14 December 2020

\begin{abstract}
Salicylic acid and jasmonic acid are two important plant hormones that trigger the plant defense responses and regulate the accumulation of bioactive compounds in plants. In the present study, the effects of salicylic acid (SA) and methyl jasmonate (MeJA) on flavonoid and carotenoid accumulation were investigated in the juice sacs of Satsuma mandarin in vitro. The results showed that SA treatment was effective to enhance the contents of eriocitrin, narirutin, poncirin, and $\beta$-cryptoxanthin in the juice sacs $(p<0.05)$. In contrast, the MeJA treatment inhibited flavonoid and carotenoid accumulation in the juice sacs $(p<0.05)$. Gene expression results showed that the changes of flavonoid and carotenoid contents in the SA and MeJA treatments were highly regulated at the transcriptional level. In addition, a transcriptional factor CitWRKY70 was identified in the microarray analysis, which was induced by the SA treatment, while suppressed by the MeJA treatment. In the SA and MeJA treatments, the change in the expression of CitWRKY70 was consistent with that of flavonoid and carotenoid biosynthetic key genes. These results indicated that CitWRKY70 might be involved in the regulation of flavonoid and carotenoid accumulation in response to SA and MeJA treatments in the juice sacs of citrus fruit.
\end{abstract}

Keywords: flavonoid; carotenoid; salicylic acid; methyl jasmonate; citrus fruit; juice sacs

\section{Introduction}

Fruit ripening is a complex developmental process involving a number of physiological and biochemical changes that are thought to be under hormonal and environmental control [1,2]. Carotenoids, whose accumulation increase during ripening in citrus, are secondary metabolites with antioxidant effects in plants and are an important group of natural pigments. In particular, $\beta$-cryptoxanthin is an important xanthophyll accumulated in fruits and vegetables. Some epidemiological studies have 
reported that dietary intake of $\beta$-cryptoxanthin reduced the risks of certain diseases, especially cancers, diabetes, and rheumatism, because of its antioxidant activity [3-8].

In citrus fruit, flavonoids are also important secondary metabolites that tend to accumulate more in peel than in the pulp of the citrus fruit [9]. Flavonoids are a group of natural pigments with antifungal and anti-insect activities in plants and are thought to be involved in pathogen defense [10,11]. In addition, flavonoids have been reported to have anticancer and anti-allergy activities in the human body [12-14]. In citrus fruit, the contents of flavonoids decreased gradually during the ripening process. To date, however, the molecular mechanism that regulates flavonoid accumulation and degradation is still unclear [11].

Salicylic acid and jasmonic acid are two important plant hormones involved in plant development and growth, and their roles in regulating carotenoid and flavonoid accumulation have been studied in different plant species. In previous studies, it was reported that salicylic acid treatment increased flavonoid content in tea and decreased carotenoid content in plum $[15,16]$. In apple peel, it was found that methyl jasmonate treatment significantly promoted $\beta$-carotene accumulation [17]. In tomato, methyl jasmonate treatment promotes the lycopene accumulation through upregulating carotenoid biosynthetic pathway gene expression [18]. In citrus fruit, the research on the effects of salicylic acid and jasmonic acid is very limited. Huang et al. reported that salicylic acid treatment induced flavonoid and carotenoid accumulation and further enhanced antioxidant activity of preharvest navel oranges [19]. To date, however, the roles of salicylic acid and jasmonic acid in regulating the carotenoid and flavonoid accumulation in the juice sacs are still far from being elucidated in citrus fruit.

In the present study, to investigate how salicylic acid (SA) and methyl jasmonate (MeJA) treatments regulate flavonoid and carotenoid accumulation in the juice sacs of citrus fruit, we set up an in vitro system [20]. In the in vitro system, the juice sacs were cultured on MS medium supplemented with SA or MeJA, and the environmental conditions, such as light, water stress, and temperature were identical among the treatments. We evaluated the effects of plant hormones (abscisic acid and gibberellin), different colors of LED lights (blue and red), water stresses (sucrose and mannitol), and temperatures on carotenoid and ascorbate accumulation in the juice sacs of citrus fruit using this in vitro system [4,20-22]. In the present study, to elucidate the regulation of carotenoid and flavonoid metabolisms in response to SA and MeJA treatments in citrus fruit, the effects of SA and MeJA on carotenoid and flavonoid accumulation and the expression of genes related to carotenoid and flavonoid biosyntheses were investigated in the juice sacs in vitro. The results presented in this study provide new strategies to promote the accumulation of flavonoid and carotenoid and improve the quality of citrus fruit.

\section{Materials and Methods}

\subsection{Plant Materials}

Immature fruit of Satsuma mandarin (Citrus unshiu Marc.) were harvested from the Fujieda Farm of Shizuoka University (Shizuoka, Japan) during July 2016, and used as the plant materials.

\subsection{In Vitro Culture System and Treatment}

The fruits were surface sterilized by the following procedure: (1) rinsing the fruit in $70 \%$ ethanol, (2) soaking then in $1 \%(w / v) ~ N a O C l$ for $1 \mathrm{~h}$, and (3) rinsing the fruit with sterile water. After surface sterilization, juice sacs were excised and placed randomly on $10 \mathrm{~mL}$ of Murashige and Skoog (MS) medium supplemented with $1 \%(w / v)$ agar and $10 \%(w / v)$ sucrose [20] for two weeks at $20{ }^{\circ} \mathrm{C}$ in the dark. After that, the juice sacs were transplanted to a new MS medium supplemented with $1 \%(w / v)$ agar, $10 \%$ $(w / v)$ sucrose, and $10 \mu \mathrm{M} \mathrm{SA}$ or MeJA, and then were cultured for 24,48 , and $72 \mathrm{~h}$. Juice sacs cultured in the MS medium without plant hormones were used as a control. Finally, for each trial, the juice sacs were collected and immediately frozen in liquid nitrogen. The samples were kept at $-80^{\circ} \mathrm{C}$ until use. 


\subsection{Extraction and Determination of Flavonoids}

The extraction and determination of flavonoids in citrus juice sacs were conducted using the methods described previously [23]. Flavonoids were extracted from the freeze-dried juice sacs using a methanol/dimethyl sulfoxide $(50: 50, v / v)$ extraction solution. The supernatant obtained by centrifugation $(21,500 \times g$ for $10 \mathrm{~min})$ was filtered through a $0.22 \mu \mathrm{m}$ syringe filter (Shimadzu GLC, Tokyo, Japan), and the filtrate was analyzed by using a reverse-phase HPLC system (Jasco, Tokyo, Japan) fitted with a YMC-UltraHT pro C18 column (Waters, Milford, MA, USA). In this study, eriocitrin, narirutin, hesperidin, and poncirin were detected at $274 \mathrm{~nm}$, and rhoifolin was detected at $338 \mathrm{~nm}$ (Supplementary Materials, Figure S1a,b). The content was estimated by the standard curves and expressed as $\mathrm{mg} \mathrm{g}^{-1}$ of dry weight. The total flavonoids were calculated by summing all identified flavonoids. Flavonoid quantification was performed in three replicates.

\subsection{Extraction and Determination of Carotenoids}

The extraction and determination of carotenoids in citrus juice sacs were conducted using the methods described previously [24]. Pigments were extracted from the juice sacs using a hexane/ acetone/ethanol $(50: 25: 25, v / v)$ as extraction solvent containing $10 \%(w / v)$ magnesium carbonate basic. The organic solvents were completely evaporated under vacuum by rotary evaporator at maximum $35^{\circ} \mathrm{C}$. Afterwards, the extracts were saponified with $8 \mathrm{~mL} \mathrm{20 \% (w/v)} \mathrm{methanolic} \mathrm{KOH}$ and $12 \mathrm{~mL}$ diethyl ether containing $0.1 \%(w / v)$ 2,6-di-tert-butyl-4-methylphenol. Then, water-soluble extracts were removed by adding $\mathrm{NaCl}$-saturated water. The pigments, repartitioned into the diethyl ether phase, were recovered and evaporated to dryness. Subsequently, the residue was redissolved in $5 \mathrm{~mL}$ of a tert-butyl-methyl-ether (TBME)/methanol $(1: 1, v / v)$ solution. After filtered through a $0.22 \mu \mathrm{m}$ syringe filter (Shimadzu GLC, Tokyo, Japan), $1 \mathrm{~mL}$ of solvent were completely evaporated and redissolved in $100 \mu \mathrm{L}$ TBME/methanol $(1: 1, v / v)$ solution. The samples were analyzed by using a reverse-phase HPLC system (Jasco, Tokyo, Japan) fitted with a YMC Carotenoid S- 5 column (Waters, Milford, MA). In this study, all-trans-violaxanthin, 9-cis-violaxanthin, lutein, and $\beta$-cryptoxanthin, and $\beta$-carotene were detected at $452 \mathrm{~nm}$ (Supplementary Materials, Figure S1c). The contents of $\beta$-carotene, $\beta$-cryptoxanthin, lutein, all-trans-violaxanthin, and 9-cis-violaxanthin were estimated by the standard curves and expressed as $\mu \mathrm{g} \mathrm{g}^{-1}$ of fresh weight [24]. The total carotenoids were calculated by summing all identified carotenoids. Carotenoid quantification was performed in three replicates.

\subsection{Analysis of Gene Expression by Using Real-Time Quantitative RT-PCR}

Total RNA was extracted from the juice sacs according to a previously reported method [25]. The total RNA was cleaned up with the RNeasy Mini kit (Qiagen, Hilden, Germany) with on-column DNase digestion. The reactions of reverse transcription (RT) were performed with $2 \mu \mathrm{g}$ of purified RNA, a random hexamer, and TaqMan Reverse Transcription Reagents at $37^{\circ} \mathrm{C}$ (Applied Biosystems, Foster City, CA, USA).

In Satsuma mandarin, the sequences of CitUFGT2 and CitWRKY70 have not been reported, thus, in the present study, we first amplified the cDNAs of CitUFGT2 and CitWRKY70 by PCR, and then the samples were sequenced by Fasmac Co., Ltd. TaqMan MGB probes and sets of primers for flavonoid biosynthetic genes (CitCHS1, CitCHS2, CitCHI, CitUFGT2, and CitF3' H), carotenoid biosynthetic genes (CitPSY, CitPDS, CitLCYb1, CitLCYe, CitHYb, and CitNCED3), and transcription factor gene (CitWRKY70) were designed with the Primer Express software (Supplementary Materials, Table S1). TaqMan real-time PCR was conducted with the TaqMan Universal PCR Master Mix (Applied Biosystems) using a StepOnePlus ${ }^{\mathrm{TM}}$ Real-Time PCR System (Applied Biosystems). Each reaction mixture contained template cDNA, a TaqMan MGB Probe $(250 \mathrm{nM})$, and primers $(900 \mathrm{nM})$. The thermal cycling conditions consisted of $95^{\circ} \mathrm{C}$ for $10 \mathrm{~min}$, followed by 40 cycles of $95^{\circ} \mathrm{C}$ for $15 \mathrm{~s}$, and $60^{\circ} \mathrm{C}$ for $1 \mathrm{~min}$. Real-time quantitative RT-PCR was performed in three replicates for each sample. 


\subsection{RNA Isolation and Fluorescent Labeling of Probes}

Total RNA was extracted by the methods of Ikoma et al. [25] from the juice sacs at 24, 48, and $72 \mathrm{~h}$, after SA or methyl MeJA treatment or control. At least three independent RNA extractions were used in probe labeling for experimental reproducibility. The total RNA ( $400 \mathrm{ng}$ ) of all samples was labeled with the fluorescence Cy5, while the untreated $0 \mathrm{~h}$ sample was labeled with Cy3 according to the instructions for the Low RNA input linear amplification and labeling kit (Agilent Technologies, Palo Alto, CA, USA). Labeled cRNA was purified using the Qiagen RNeasy mini kit (Qiagen, Hilden, Germany). Hybridization and washing were performed according to the Agilent's instructions. Glass slides were hybridized overnight at $60^{\circ} \mathrm{C}$ in a hybridization buffer containing a fragment of Cy3-or Cy5-labeled cRNA. After hybridization, slides were washed in $6 \times$ SSC, $0.005 \%$ Triton X-100 for $10 \mathrm{~min}$ at room temperature and $0.1 \times$ SSC, $0.005 \%$ Triton X-100 for $5 \mathrm{~min}$ at $4{ }^{\circ} \mathrm{C}$. After drying the slides with gaseous nitrogen, hybridized slides were scanned with the use of an Agilent microarray scanner (Agilent Technologies, Palo Alto, CA, USA). The intensities of the Cy5 and Cy3 fluorescent signals from each spot were automatically normalized, and the ratio value (Cy5/Cy3) was calculated using Feature Extraction version 7.1 software (Linear \& LOWESS analysis, Agilent). Data analysis was carried out using Subio (Subio).

\subsection{Statistical Analysis}

All values are shown as the mean \pm SE. The data were analyzed, and Tukey's HSD test was used to analyze the differences amongst the treatments at $p<0.05$ level.

\section{Results}

\subsection{Effect of Salicylic Acid (SA) and Methyl Jasmonate (MeJA) on Flavonoid Content in Citrus Juice Sacs} In Vitro

In this study, five flavonoids, eriocitrin, narirutin, hesperidin, poncirin, and rhoifolin were detected in juice sacs of Satsuma mandarin (Supplementary Materials, Figure S1a,b). In the juice sacs, hesperidin is the most predominantly accumulated, followed by narirutin. As compared with hesperidin and narirutin, the contents of eriocitrin, poncirin, and rhoifolin are relatively low in the juice sacs. To investigate how SA and MeJA treatments regulate flavonoid accumulation in the juice sacs of Satsuma mandarin, the changes in the contents of eriocitrin, narirutin, hesperidin, poncirin, and rhoifolin were determined in the juice sacs in vitro. As shown in Figure 1, significant changes in the flavonoid contents were observed after 72-h treatments of SA and MeJA. The content of rhoifolin was decreased, while the contents of eriocitrin, narirutin, and poncirin were significantly increased by the SA treatment, which were 0.6-fold, 0.3-fold, and 0.2-fold higher than that of the control, respectively. Therefore, the total flavonoid content after the SA treatment was higher than that of the control (111.8 vs. $\left.99.2 \mathrm{mg} \cdot \mathrm{g}^{-1}\right)$. Conversely, the contents of hesperidin, poncirin, and rhoifolin were significantly reduced after the MeJA treatment, and as a result the total flavonoid content was lower than that of the control.

\subsection{Effect of SA and MeJA on Carotenoid Content in Citrus Juice Sacs In Vitro}

The effects of SA and MeJA on the accumulation of the major carotenoids in the citrus juice sacs were also investigated. Although significant change in the total carotenoid content was not observed, the accumulation of individual carotenoid, such as $\beta$-cryptoxanthin and all-trans-violaxanthin, was affected by the SA treatment (Figure 2). Indeed, the content of all-trans-violaxanthin decreased, while that of $\beta$-cryptoxanthin, which accounts for about $30 \%$ of total carotenoid, increased after the SA treatment. On the contrary, the total carotenoid content was much lower than the control after the MeJA treatment. In particular, only the content of $\beta$-cryptoxanthin increased up to 0.24 -fold with respect to the control; whilst the concentration of all-trans-violaxanthin, 9-cis-violaxanthin, and lutein significantly decreased (Figure 2). 

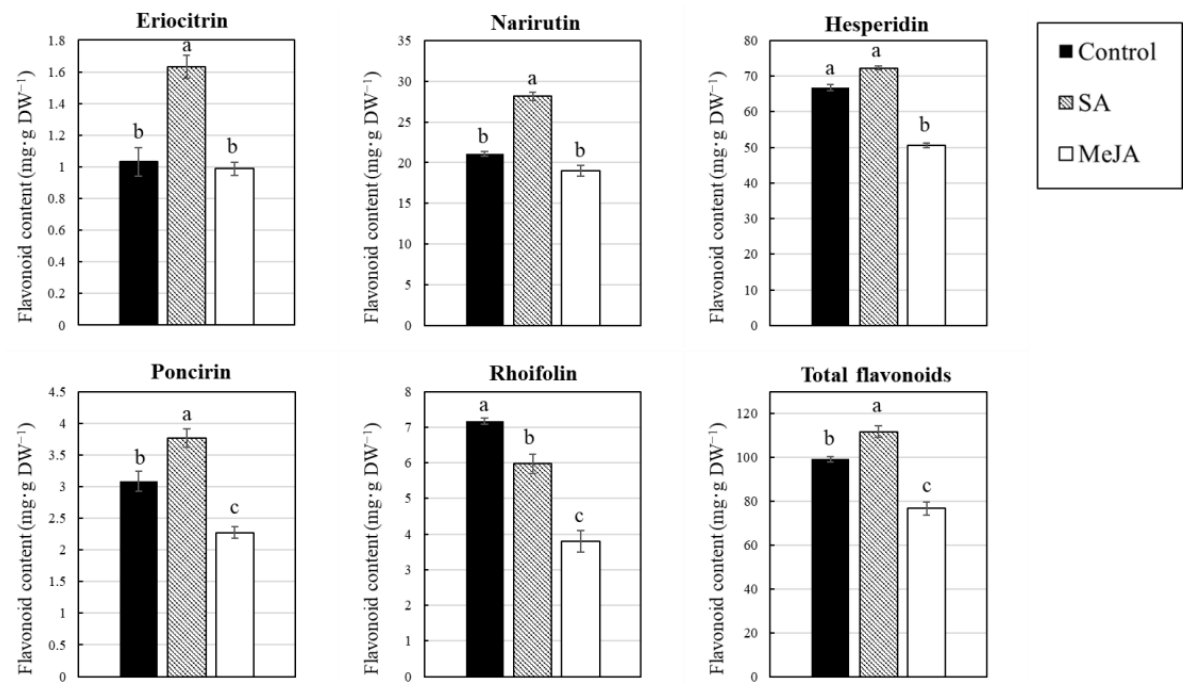

Figure 1. Effect of salicylic acid (SA) and methyl jasmonate (MeJA) treatments on the flavonoid content in the juice sacs of Satsuma mandarin in vitro. The total flavonoid was calculated by summing eriocitrin, narirutin, hesperidin, poncirin, and rhoifolin. Columns and bars represent the means and SE $(n=3)$, respectively. Different letters indicate significant differences at the 5\% level by Tukey's HSD test.
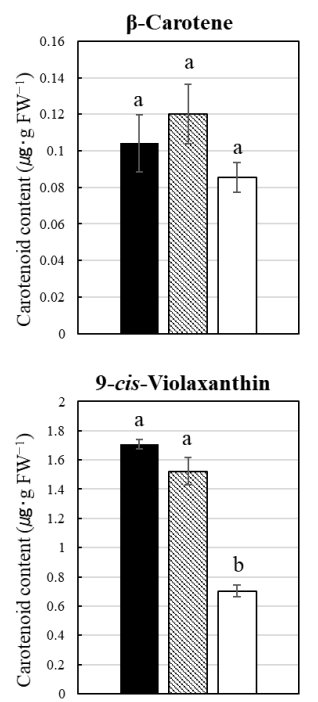
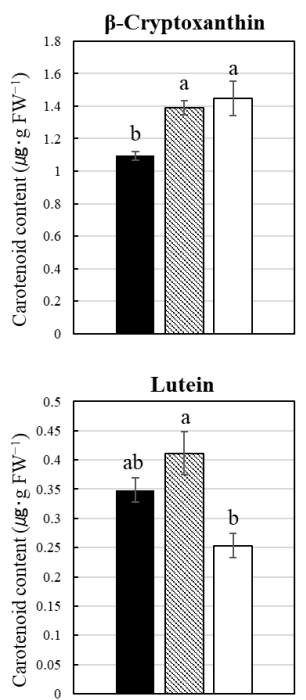
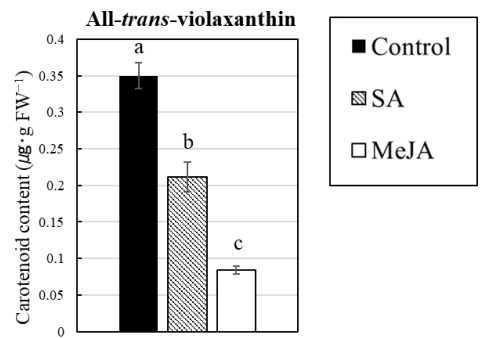

Figure 2. Effect of salicylic acid (SA) and methyl jasmonate (MeJA) treatments on the carotenoid content in the juice sacs of Satsuma mandarin in vitro. The total carotenoid was calculated by summing $\beta$-carotene, $\beta$-cryptoxanthin, all-trans-violaxanthin, 9-cis-violaxanthin, and lutein. Columns and bars represent the means and SE $(n=3)$, respectively. Different letters indicate significant differences at the $5 \%$ level by Tukey's HSD test.

\subsection{Effect of SA and MeJA on Expression of the Genes Related to Flavonoid and Carotenoid Accumulation in Citrus Juice Sacs}

In this study, the changes in the expression of the genes related to flavonoid and carotenoid metabolisms in response to SA and MeJA treatments were investigated by using real-time PCR. The results showed that the expression of CitCHS1 was downregulated, while the expression of CitCHS2, CitCHI, CitUFGT2, and CitF3' H was significantly upregulated by the SA treatment (Figure 3). In the MeJA treatment, the expression of CitCHS1 and CitF3'H was downregulated, while the expression of $\mathrm{CitCHI}$ and CitUFGT2 was upregulated in the citrus juice sacs (Figure 3). Furthermore, the expression of five carotenoid biosynthetic genes (CitPSY, CitPDS CitLCYe, CitLCYb1 and CitHYb) 
and one carotenoid catabolic gene (CitNCED3) was investigated in the citrus juice sacs. In the SA treatment, the expression of CitPSY, CitPDS, and CitHYb was upregulated, while that of CitNCED3 was downregulated. Instead, in the MeJA treatment, the expression of CitHYb was downregulated, and that of the other carotenoid metabolic genes was not significantly affected (Figure 4).
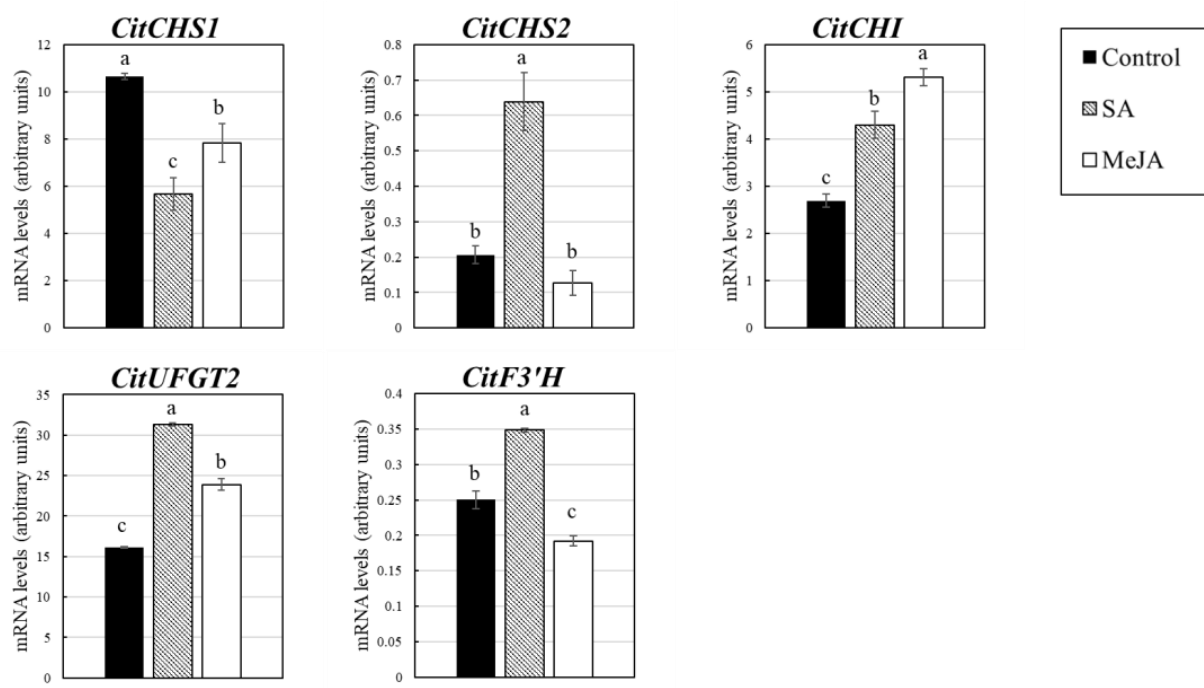

Figure 3. Effect of salicylic acid (SA) and methyl jasmonate (MeJA) treatments on the expression of flavonoid metabolism related genes in the juice sacs of Satsuma mandarin in vitro. The mRNA levels were analyzed by TaqMan real-time RT-PCR, and the expression of $18 \mathrm{~S}$ ribosomal RNA (rRNA) was used as a control to normalize the raw data. Columns and bars represent the means and SE $(n=3)$, respectively. Different letters indicate significant differences at the $5 \%$ level by Tukey's HSD test. CHS1, chalcone synthase1; CHS2, chalcone synthase2; CHI, chalcone isomerase; UFGT2, flavanone 7-O-glucosyltransferase2; F3'H, flavonoid-3'-hydroxylase.
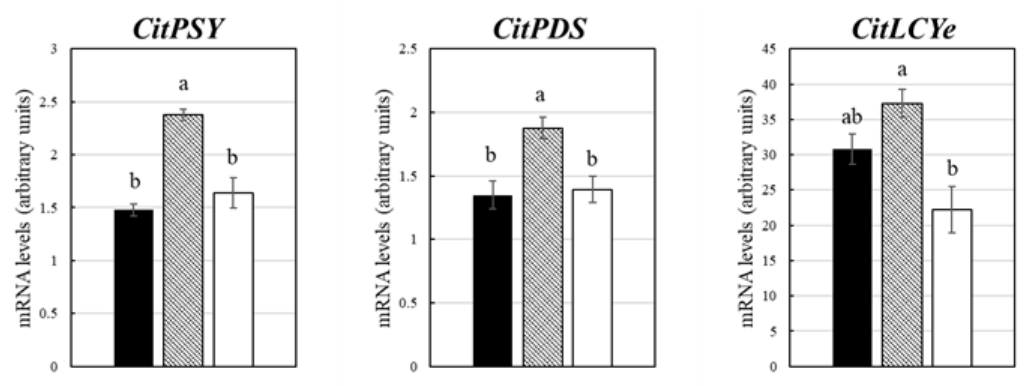

$$
\begin{aligned}
& \text { - Control } \\
& \triangle \mathrm{SA}
\end{aligned}
$$
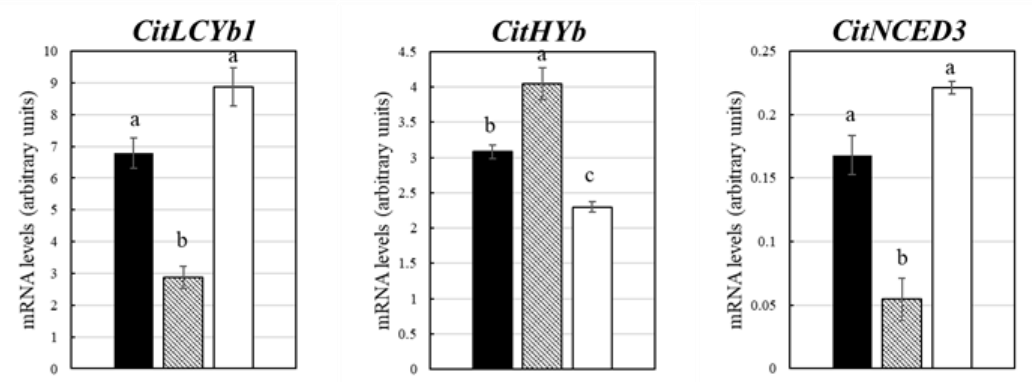

Figure 4. Effect of salicylic acid (SA) and methyl jasmonate (MeJA) treatments on the expression of carotenoid metabolism related genes in the juice sacs of Satsuma mandarin in vitro. The mRNA levels were analyzed by TaqMan real-time RT-PCR, and the expression of $18 \mathrm{~S}$ ribosomal RNA (rRNA) was used as a control to normalize the raw data. Columns and bars represent the means and SE $(n=3)$, respectively. Different letters indicate significant differences at the 5\% level by Tukey's HSD test. PSY, phytoene synthase; PDS, 15-cis-phytoene desaturase; LCYe, lycopene epsilon-cyclase; LCYb1, lycopene beta-cyclase1; HYb, beta-carotene 3-hydroxylase; NCED3, 9-cis-epoxycarotenoid dioxygenase3. 


\subsection{Identification of Transcription Factor in Response to Salicylic Acid and Methyl Jasmonate Treatments}

In the present study, differentially expressed genes were investigated in the SA and MeJA treatments by using microarray analysis. As compared with the control, the expressions of 1616 genes were found to be significantly changed after 24-, 48-, and 72-h treatments of SA (Figure 5a and Supplementary Materials, Table S2). In particular, the expression of 1286 genes was upregulated, while the expression of 330 genes was downregulated by the SA treatment. In the MeJA treatment, there were 4914 genes that were differentially expressed as compared with the control (Figure $5 b$ and Supplementary Materials, Table S3). Among them, the expression of 4404 genes was upregulated, while the expression of 510 genes was downregulated by the MeJA treatment. In this study, we found that SA treatment upregulated the key genes related to flavonoid and carotenoid biosynthesis (e.g., $C i t C H S 2$, $C i t F 3^{\prime} H$, and $C i t H Y b$ ) in the juice sacs in vitro. In contrast, the expression of these key genes was downregulated by the MeJA treatment in the juice sacs in vitro. To identify the transcriptional factors that might be involved in the regulation of flavonoid and carotenoid accumulation, we further screened the genes which were upregulated in the SA treatment while downregulated in the MeJA. As shown in Figure 6, we found that there were three genes that were upregulated by SA treatment while downregulated by the MeJA treatment (Cicev10012055m, Ciclev10022863m, and Ciclev10025958m; Table 1). Among them, a WRKY DNA-binding protein 70 was identified (Cicev10012055m), which exhibited $45.6 \%$ homology to Arabidopsis WRKY70. Expression analysis of CitWRKY70 by using real-time PCR confirmed that it was a 9.4-fold upregulated by the SA treatment, and a 14.4-fold downregulated by the MeJA treatment as compared with the control (Figure 7).

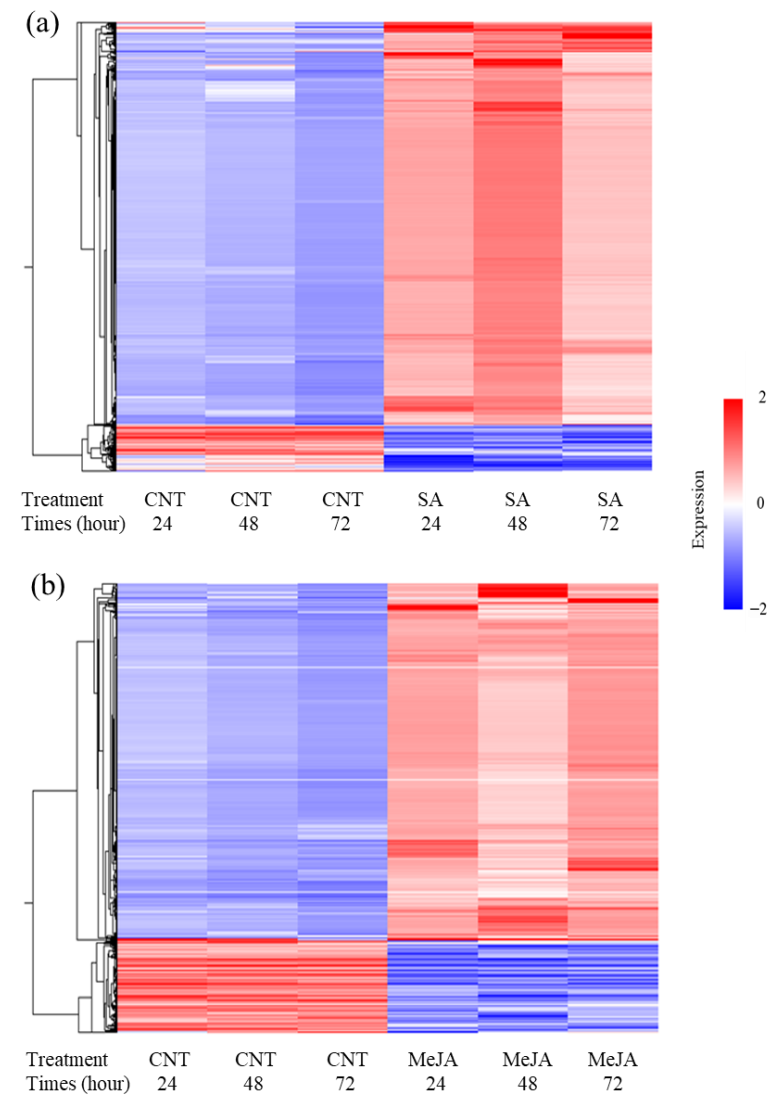

Figure 5. Hierarchical cluster analysis of 1616 salicylic acid-responsive genes and 4914 methyl jasmonate-responsive genes with more than 2-fold expression changes between salicylic acid or methyl jasmonate and control treatments. (a) Salicylic acid (SA)/control (CNT) signal intensity ratio; (b) Methyl jasmonate (MeJA)/control (CNT) signal intensity ratio. The color scale indicates a signal intensity of each gene. Tree at the left side of the matrix represents gene relationship. 
(a)

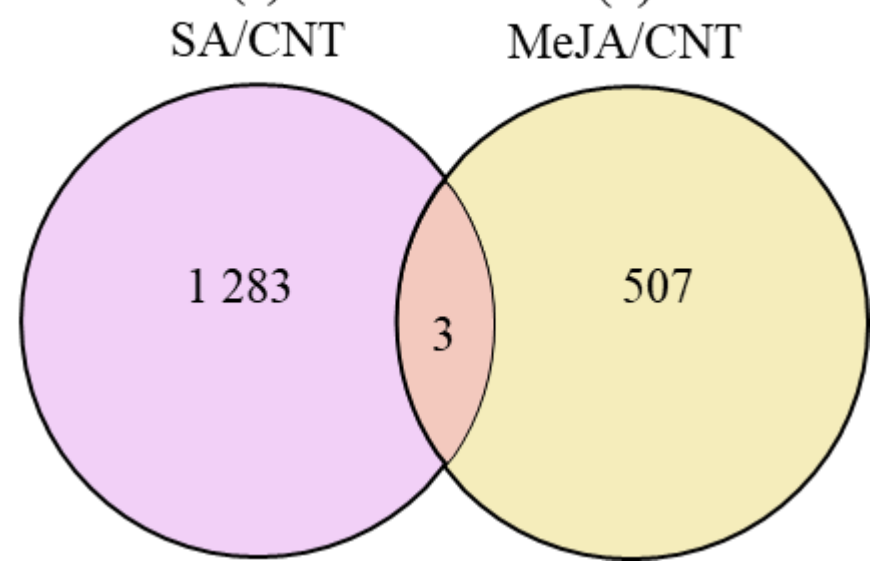

Figure 6. Two-set Venn diagram analysis of salicylic acid (SA) and methyl jasmonate (MeJA)-responsive genes in the juice sacs in vitro. (a) A set of 1286 salicylic acid-responsive genes whose expression is more than 2-fold increased by salicylic acid treatment; (b) A set of 510 methyl jasmonate-responsive genes whose expression is more than 2 -fold decreased by methyl jasmonate treatment. CNT, control.
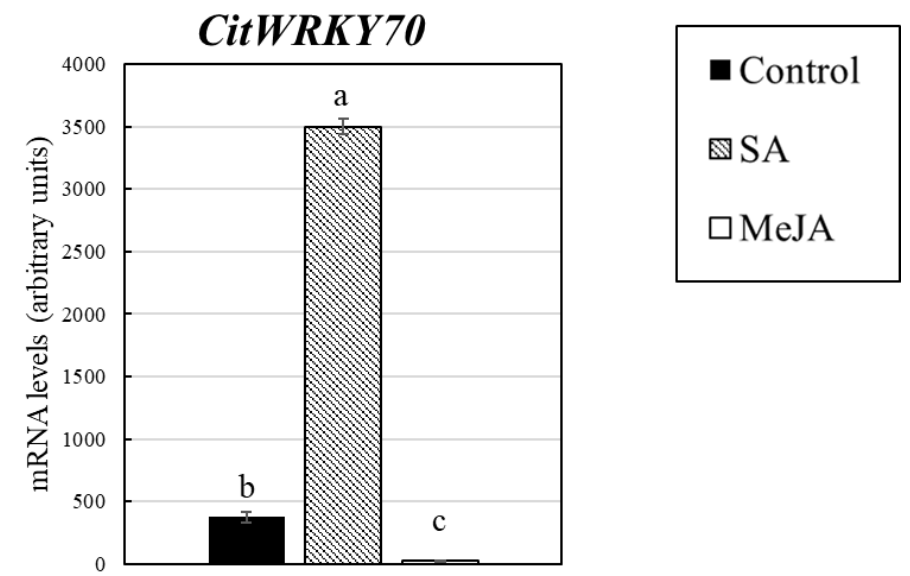

Figure 7. Effects of salicylic acid (SA) and methyl jasmonate (MeJA) treatments on the expression of CitWRKY70 in the juice sacs of Satsuma mandarin in vitro. The mRNA levels were analyzed by TaqMan real-time RT-PCR, and the expression of $18 \mathrm{~S}$ ribosomal RNA (rRNA) was used as a control to normalize the raw data. Columns and bars represent the means and SE $(n=3)$, respectively. Different letters indicate significant differences at the $5 \%$ level by Tukey's HSD test. WRKY70, WRKY DNA-binding protein 70 .

Table 1. Details of three genes that were more than 2-fold upregulated in salicylic acid (SA) treatment and 2-fold downregulated in methyl jasmonate (MeJA) treatment. CNT, control.

\begin{tabular}{|c|c|c|c|c|c|c|c|c|c|c|}
\hline \multirow{2}{*}{ ID } & \multirow{2}{*}{ mRNA } & \multirow{2}{*}{ JGI_AGI } & \multirow{2}{*}{ Abbreviations } & \multirow{2}{*}{ Enzyme } & \multicolumn{3}{|c|}{ SA/CNT } & \multicolumn{3}{|c|}{ MeJA/CNT } \\
\hline & & & & & $24 \mathrm{~h}$ & $48 \mathrm{~h}$ & $72 \mathrm{~h}$ & $24 \mathrm{~h}$ & $48 \mathrm{~h}$ & $72 \mathrm{~h}$ \\
\hline C22820 & Ciclev10022863m & & & $\begin{array}{c}\text { PROTEIN } \\
\text { NIM1-INTERACTING } 1\end{array}$ & 2.53 & 2.26 & 4.18 & 0.31 & 0.42 & 0.35 \\
\hline C25898 & Ciclev10025958m & AT4G10490.1 & DMR-6 & $\begin{array}{l}\text { 2-oxoglutarate (2OG) } \\
\text { and Fe (II)-dependent }\end{array}$ & 17.93 & 12.21 & 67.54 & 0.23 & 0.12 & 0.10 \\
\hline C12031 & Ciclev10012055m & AT3G56400.1 & WRKY70 & $\begin{array}{l}\text { WRKY DNA-binding } \\
\text { protein } 70\end{array}$ & 6.52 & 5.51 & 10.30 & 0.30 & 0.16 & 0.28 \\
\hline
\end{tabular}




\section{Discussion}

\subsection{Effect of SA on the Flavonoid and Carotenoid Accumulation in Citrus Juice Sacs In Vitro}

Flavonoids and carotenoids are two important secondary metabolites that accumulate in plants. To date, the role of SA in flavonoid accumulation has been investigated in different plant species such as tea and cucumber $[15,26]$. SA and flavonoids are both phenylpropanoid derivatives, and recent studies have suggested the SA treatment affected the flavonoid biosynthesis in plants [27]. In tea, it was reported that exogenous methyl salicylate treatment increased the expression of flavonoid biosynthesis-related genes, such as $\mathrm{CHS}$ and $\mathrm{CHI}$, and as a result the total content of flavonoid was enhanced by the methyl salicylate treatment [15]. In cucumber, $0.5 \mathrm{mM}$ salicylic acid treatment was found to be effective to enhance the contents of total phenolic compound and total flavonoid [26]. In the present study, the results showed that SA treatment induced the accumulation of eriocitrin, narirutin, and poncirin in the citrus juice sacs in vitro (Figure 1). The gene expression analysis showed that the expression of CitCHS2, CitUFGT2, and CitF3'H was markedly increased by the SA treatment (Figure 3). As previously reported, CitCHS2, which was highly expressed in the young fruit, was an important gene regulating the biosynthesis of flavonoid in citrus fruit [28]. In the present study, the high expression of CitCHS2 could lead to enhance the contents of eriocitrin, narirutin, hesperidin, and poncirin in the SA-treated juice sacs. In addition, $\mathrm{CitF}^{\prime} \mathrm{H}$, which catalyzes the hydroxylation of $\beta$-rings at the $3^{\prime}$-position, is a rate limiting enzyme for flavonoid biosynthesis in plant [29]. In this study, the high expression of CitF3'H in the SA treatment could contribute to increasing the content of eriocitrin, which has a hydroxy group on the $\beta$-rings at the 3 '-position, in the citrus juice sacs.

Different from flavonoid, the roles of SA in carotenoid accumulation were controversial in plants. In wheat and moong seedings, total carotenoid content, size of xanthophyll pool, and de-epoxidation rate were induced along with an increase in SA concentration [30]. In plum, in contrast, the preharvest treatment of salicylate significantly reduced the total carotenoid content [16]. In the present study, the results showed that the total carotenoid content was not significantly affected by the SA treatment, but the content of $\beta$-cryptoxanthin, which was the major carotenoid accumulated in the juice sacs, was clearly enhanced by the SA treatment. The gene expression results suggested that the higher expression of carotenoid biosynthetic genes, CitPSY, CitPDS, and CitHYb and lower expression of carotenoid catabolic gene CitNCED3 could lead to enhance $\beta$-cryptoxanthin content in the juice sacs treated by SA.

Summarizing, we found that SA treatment was effective to enhance the contents of eriocitrin, narirutin, and poncirin and $\beta$-cryptoxanthin in the citrus juice sacs of Satsuma mandarin. Overall, our findings were consistent with a previous study, in which the preharvest treatment of salicylate caused an increase of carotenoids and flavonoids contents in navel oranges [19]. Flavonoids and carotenoids are important antioxidant compounds in citrus fruits with beneficial effects to human health [31]. Thus, the results presented in this study suggested that SA treatment could be an effective method to improve the quality and nutritional values of citrus fruit.

\subsection{Effect of MeJA on the Flavonoid and Carotenoid Accumulation in Citrus Juice Sacs In Vitro}

Jasmonic acid is a plant hormone that acts as a signal of plant responses to biotic and abiotic stresses, including the regulation of the bioactive compounds accumulation in plants [32]. It has been reported that MeJA was effective to induce flavonoid accumulation in apples [33], red Chinese pear [34], red raspberry [35], and blackberry [36]. To date, the studies of MeJA-regulated flavonoid accumulation have been mainly focused on anthocyanin and phenolic biosyntheses, while its effects on the flavonoids that are specifically accumulated in the citrus fruit have been largely unknown. In the present study, the results showed that the MeJA treatment significantly decreased the contents of hesperidin, poncirin, and rhoifolin in the juice sacs of citrus fruit, and as a result the total flavonoid content was much lower than that of the control. The gene expression results showed the downregulation of expression of CitCHS1, CitCHS2, and CitF3' H was consistent with the decrease in the flavonoids 
contents in the juice sacs. In addition, we found that the MeJA treatment not only inhibited flavonoid accumulation, but also caused a significant decrease of carotenoids in the juice sacs. Indeed, the contents of all-trans-violaxanthin, 9-cis-violaxanthin, lutein, as well as total carotenoid were much lower than that of the control. Moreover, the expression of $\mathrm{CitHYb}$, which is a key gene responsible for xanthophyll biosynthesis, was downregulated by the MeJA treatment. Recently, the roles of jasmonate on carotenoid accumulation has been reported in lettuce, tomato, and maize kernels, in which it was indicated that jasmonate positively regulated the accumulation of lycopene and lutein, and a deficiency of jasmonate led to a great reduction of lycopene in tomato fruit $[18,37,38]$. However, the effect of jasmonate on carotenoids accumulation was dose dependent, i.e., when the concentration of MeJA was higher than $0.5 \mu \mathrm{M}$, the carotenoid biosynthesis was inhibited in tomato and maize kernels. In the present study, we treated the juice sacs with $10 \mu \mathrm{M}$ MeJA and found that the carotenoids content was significantly decreased in the juice sacs. These results were in agreement with previous studies in tomato and maize kernels [38]. In future studies, research on the effects of different concentrations of MeJA on carotenoid biosynthesis is expected, which should contribute to improving the carotenoid accumulation in the juice sacs of citrus fruit.

\subsection{Identification of Transcription Factor in Response to SA and MeJA Treatments}

WRKY70 is a unique member of the WRKY transcriptional factor family controlling plant defense, senescence, and developmental processes [39-43]. Li et al. [44] reported that WRKY70 was a common component in salicylic acid and jasmonic acid mediated signal pathways, and played a pivotal role in determining the balance between salicylic acid-dependent and jasmonic acid-dependent defense pathway. In Arabidopsis, it was found that the expression of WRKY70 was activated by SA and repressed by MeJA. In citrus, however, the research on WRKY70 has not been reported. In the present study, differentially expressed genes in the SA and MeJA treatments were investigated by using microarray analysis. We found that the expression of CitWRKY70 was induced by the SA treatment, while suppressed by the MeJA treatment in the juice sacs in vitro. In our study, it was showed that the expression of key genes related to flavonoid and carotenoid biosyntheses (e.g., CitCHS2, CitF3'H, and $C i t H Y b$ ) was also induced by SA treatment, while suppressed by the MeJA treatment. Thus, it was deduced that CitWRKY70 could be involved in the regulation of flavonoid and carotenoid accumulation in response to the treatments with the two phytohormones. In the future, more in-depth researches on the function of WRKY70 in flavonoid and carotenoid biosyntheses are still needed, which would contribute to elucidating the regulation mechanisms of flavonoid and carotenoid accumulation in response to SA and MeJA treatments in citrus fruit.

\section{Conclusions}

In the present study, the effects of SA and MeJA on flavonoid and carotenoid accumulation were investigated in the juice sacs of Satsuma mandarin in vitro. The results suggested that the plant hormones SA and MeJA are important factors regulating flavonoid and carotenoid accumulation in the citrus juice sacs. The SA treatment was effective to enhance the contents of eriocitrin, narirutin, poncirin, and $\beta$-cryptoxanthin in the citrus juice sacs. Whereas the carotenoid and flavonoid accumulation was inhibited by the MeJA treatment in the citrus juice sacs in vitro. The gene expression results suggested that the changes in the flavonoid and carotenoid accumulation in the SA and MeJA treatments were highly regulated at the transcriptional level. In addition, a transcriptional factor CitWRKY70 was identified in this study by using the microarray analysis. The changes in the expression of CitWRKY70 was consistent with those of the key flavonoid and carotenoid biosynthetic genes, indicating that CitWRKY70 could be involved in the regulation of flavonoid and carotenoid accumulation in response to SA and MeJA treatments. In this study, the regulation of flavonoid and carotenoid accumulation by SA and MeJA treatments were investigated, and their molecular mechanisms were deeply discussed in citrus fruit. The results present herein contribute to elucidating the roles of SA and MeJA in plants and provide new strategies to modify flavonoid and carotenoid accumulation in citrus fruit. Moreover, 
as the flavonoid and carotenoid contents were significantly enhanced by the SA treatment in this study, it was indicated that application of SA could be an effective method to improve the nutritional and commotional values of citrus fruit. In the future study, more in-depth researches on the function of WRKY70 and effects of different concentrations of MeJA in citrus fruit are expected, which would contribute to further elucidating the regulation mechanisms of flavonoid and carotenoid accumulation in response to SA and MeJA treatments in citrus fruit.

Supplementary Materials: The following are available online at http://www.mdpi.com/2076-3417/10/24/8916/s1, Table S1: Primer sequences and TaqMan MGB Probes used for the TaqMan real-time RT-PCR of the genes related to plant hormones, flavonoid and carotenoid metabolisms., Table S2: Representative salicylic acid responsive genes showing differential expression ( $>2$-fold) between salicylic acid and control treatments (salicylic acid/ control ratio). Table S3: Representative methyl jasmonate responsive genes showing differential expression ( $>2$-fold) between methyl jasmonate and control treatments (methyl jasmonate/control ratio). Figure S1: Chromatograms in the flavonoid and carotenoid in the juice sacs of citrus fruit in vitro.

Author Contributions: Conceptualization, G.M. and M.K.; methodology, T.S., H.F., and T.E.; validation, R.Y., G.M., L.Z., and M.K.; formal analysis, R.Y. and G.M.; investigation, R.Y., M.H., and G.M.; resources, M.Y. and K.Y.; data curation, R.Y., G.M., L.Z., and M.K.; writing—original draft preparation, R.Y. and G.M.; writing一review and editing, L.Z. and M.K.; visualization R.Y., G.M., and L.Z.; supervision, M.K.; project administration, G.M. and M.K.; funding acquisition, G.M. and M.K. All authors have read and agreed to the published version of the manuscript.

Funding: This work was supported by KAKENHI grant numbers JP26292015 (to M.K.) and JP19K06030 (to G.M.) from Japan Society for the Promotion of Science (JSPS).

Conflicts of Interest: The authors declare no conflict of interest.

\section{References}

1. Fujii, H.; Shimada, T.; Sugiyama, A.; Nishikawa, F.; Endo, T.; Nakano, M.; Ikoma, Y.; Shimizu, T.; Omura, M. Profiling ethylene-responsive genes in mature mandarin fruit using a citrus 22K oligoarray. Plant Sci. 2007, 173, 340-348. [CrossRef]

2. Giovannoni, J.J. Genetic Regulation of Fruit Development and Ripening. Plant Cell 2004, 16 (Suppl. 1), S170-S180. [CrossRef]

3. Takayanagi, K.; Morimoto, S.; Shirakura, Y.; Mukai, K.; Sugiyama, T.; Tokuji, Y.; Ohnishi, M. Mechanism of Visceral Fat Reduction in Tsumura Suzuki Obese, Diabetes (TSOD) Mice Orally Administered $\beta$-Cryptoxanthin from Satsuma Mandarin Oranges (Citrus Unshiu Marc). J. Agric. Food Chem. 2011, 59, 12342-12351. [CrossRef]

4. Ma, G.; Zhang, L.; Iida, K.; Madono, Y.; Yungyuen, W.; Yahata, M.; Yamawaki, K.; Kato, M. Identification and quantitative analysis of $\beta$-cryptoxanthin and $\beta$-citraurin esters in satsuma mandarin fruit during the ripening process. Food Chem. 2017, 234, 356-364. [CrossRef]

5. Yamaguchi, M. Role of carotenoid $\beta$-cryptoxanthin in bone homeostasis. J. Biomed. Sci. 2012, 19, 1-13. [CrossRef] [PubMed]

6. Iskandar, A.R.; Liu, C.; Smith, D.E.; Hu, K.-Q.; Choi, S.-W.; Ausman, L.M.; Wang, X.-D. $\beta$-Cryptoxanthin Restores Nicotine-Reduced Lung SIRT1 to Normal Levels and Inhibits Nicotine-Promoted Lung Tumorigenesis and Emphysema in A/J Mice. Cancer Prev. Res. 2013, 6, 309-320. [CrossRef] [PubMed]

7. Sugiura, M.; Nakamura, M.; Ogawa, K.; Ikoma, Y.; Ando, F.; Shimokata, H.; Yano, M. Dietary patterns of antioxidant vitamin and carotenoid intake associated with bone mineral density: Findings from Post-Menopausal Japanese Female Subjects. Osteoporos. Int. 2011, 22, 143-152. [CrossRef] [PubMed]

8. Ma, G.; Zhang, L.; Sugiura, M.; Kato, M. Citrus and health. In The Genus Citrus; Elsevier: Amsterdam, The Netherlands, 2020; pp. 495-511.

9. Nogata, Y.; Sakamoto, K.; Shiratsuchi, H.; Ishii, T.; YANO, M.; Ohta, H. Flavonoid Composition of Fruit Tissues of Citrus Species. Biosci. Biotechnol. Biochem. 2006, 70, 178-192. [CrossRef] [PubMed]

10. Kim, B.-G.; Kim, H.; Hur, H.-G.; Lim, Y.; Ahn, J.-H. Regioselectivity of 7-O-methyltransferase of poplar to flavones. J. Bbiotechnol. 2006, 126, 241-247. [CrossRef]

11. Napal, G.N.D.; Carpinella, M.C.; Palacios, S.M. Antifeedant activity of ethanolic extract from Flourensia oolepis and isolation of pinocembrin as its active principle compound. Bioresour. Technol. 2009, 100,3669-3673. [CrossRef] 
12. Cheng, H.-L.; Hsieh, M.-J.; Yang, J.-S.; Lin, C.-W.; Lue, K.-H.; Lu, K.-H.; Yang, S.-F. Nobiletin inhibits human osteosarcoma cells metastasis by blocking ERK and JNK-mediated MMPs expression. Oncotarget 2016, 7, 35208. [CrossRef]

13. Goh, J.X.H.; Tan, L.T.-H.; Goh, J.K.; Chan, K.G.; Pusparajah, P.; Lee, L.-H.; Goh, B.-H. Nobiletin and Derivatives: Functional Compounds from Citrus Fruit Peel for Colon Cancer Chemoprevention. Cancers 2019, 11, 867. [CrossRef] [PubMed]

14. Takano, H.; Osakabe, N.; Sanbongi, C.; Yanagisawa, R.; Inoue, K.-I.; Yasuda, A.; Natsume, M.; Baba, S.; Ichiishi, E.-I.; Yoshikawa, T. Extract of Perilla frutescens Enriched for Rosmarinic Acid, a Polyphenolic Phytochemical, Inhibits Seasonal Allergic Rhinoconjunctivitis in Humans. Exp. Biol. Med. 2004, 229, $247-254$. [CrossRef] [PubMed]

15. Li, X.; Zhang, L.-P.; Zhang, L.; Yan, P.; Ahammed, G.J.; Han, W.-Y. Methyl Salicylate Enhances Flavonoid Biosynthesis in Tea Leaves by Stimulating the Phenylpropanoid Pathway. Molecules 2019, 24, 362. [CrossRef] [PubMed]

16. Martínez-Esplá, A.; Zapata, P.J.; Valero, D.; Martínez-Romero, D.; Díaz-Mula, H.M.; Serrano, M. Preharvest treatments with salicylates enhance nutrient and antioxidant compounds in plum at harvest and after storage. J. Sci. Food Agric. 2018, 98, 2742-2750. [CrossRef] [PubMed]

17. Pérez, A.G.; Sanz, C.; Richardson, D.G.; Olías, J.M. Methyl Jasmonate Vapor Promotes $\beta$-Carotene Synthesis and Chlorophyll Degradation in Golden Delicious Apple Peel. J. Plant Growth Regul. 1993, 12, 163. [CrossRef]

18. Liu, L.; Wei, J.; Zhang, M.; Zhang, L.; Li, C.; Wang, Q. Ethylene independent induction of lycopene biosynthesis in tomato fruits by jasmonates. J. Exp. Bot. 2012, 63, 5751-5761. [CrossRef]

19. Huang, R.; Xia, R.; Lu, Y.; Hu, L.; Xu, Y. Effect of pre-harvest salicylic acid spray treatment on post-harvest antioxidant in the pulp and peel of 'Cara cara' navel orange (Citrus sinenisis L. Osbeck). J. Sci. Food Agric. 2008, 88, 229-236. [CrossRef]

20. Zhang, L.; Ma, G.; Kato, M.; Yamawaki, K.; Takagi, T.; Kiriiwa, Y.; Ikoma, Y.; Matsumoto, H.; Yoshioka, T.; Nesumi, H. Regulation of carotenoid accumulation and the expression of carotenoid metabolic genes in citrus juice sacs in vitro. J. Exp. Bot. 2012, 63, 871-886. [CrossRef]

21. Ma, G.; Zhang, L.; Yungyuen, W.; Sato, Y.; Furuya, T.; Yahata, M.; Yamawaki, K.; Kato, M. Accumulation of carotenoids in a novel citrus cultivar 'Seinannohikari' during the fruit maturation. Plant Physiol. Biochem. 2018, 129, 349-356. [CrossRef]

22. Zhang, L.; Ma, G.; Yamawaki, K.; Ikoma, Y.; Matsumoto, H.; Yoshioka, T.; Ohta, S.; Kato, M. Effect of Blue LED light intensity on carotenoid accumulation in citrus juice sacs. Physiol. Plant. 2015, 188, 58-63. [CrossRef] [PubMed]

23. Seoka, M.; Ma, G.; Zhang, L.; Yahata, M.; Yamawaki, K.; Kan, T.; Kato, M. Expression and functional analysis of the nobiletin biosynthesis-related gene CitOMT in citrus fruit. Sci. Rep. 2020, 10, 15288. [CrossRef] [PubMed]

24. Kato, M.; Ikoma, Y.; Matsumoto, H.; Sugiura, M.; Hyodo, H.; Yano, M. Accumulation of Carotenoids and Expression of Carotenoid Biosynthetic Genes during Maturation in Citrus Fruit. Physiol. Plant. 2004, 134, 824-837. [CrossRef] [PubMed]

25. Ikoma, Y.; Yano, M.; Ogawa, K.; Yoshioka, T.; Xu, Z.C.; Hisada, S.; Omura, M.; Moriguchi, T. Isolation and Evaluation of RNA from Polysaccharide-Rich Tissues in Fruit for Quality by CDNA Library Construction and RT-PCR. J. Jpn. Soc. Hort. Sci. 1996, 64, 809-814. [CrossRef]

26. Preciado-Rangel, P.; Reyes-Pérez, J.J.; Ramírez-Rodríguez, S.C.; Salas-Pérez, L.; Fortis-Hernández, M.; Murillo-Amador, B.; Troyo-Diéguez, E. Foliar Aspersion of Salicylic Acid Improves Phenolic and Flavonoid Compounds, and Also the Fruit Yield in Cucumber (Cucumis Sativus L.). Plants 2019, 8, 44. [CrossRef]

27. Gondor, O.K.; Janda, T.; Soós, V.; Pál, M.; Majláth, I.; Adak, M.K.; Balázs, E.; Szalai, G. Salicylic Acid Induction of Flavonoid Biosynthesis Pathways in Wheat Varies by Treatment. Front. Plant Sci. 2016, 7, 1447. [CrossRef]

28. Moriguchi, T.; Kita, M.; Tomono, Y.; Endo-Inagaki, T.; Omura, M. Gene expression in flavonoid biosynthesis: Correlation with flavonoid accumulation in developing citrus fruit. Physiol Plant. 2001, 111, 66-74. [CrossRef]

29. Baba, S.A.; Ashraf, N. Functional characterization of flavonoid $3^{\prime}$-hydroxylase, CsF3' H, from Crocus sativus L: Insights into substrate specificity and role in abiotic stress. Arch. Biochem. Biophys. 2019, 667, 70-78. [CrossRef]

30. Moharekar, S.T.; Lokhande, S.D.; Hara, T.; Tanaka, R.; Tanaka, A.; Chavan, P.D. Effect of salicylic acid on chlorophyll and carotenoid contents of wheat and moong seedlings. Photosynthetica 2003, 41, 315. [CrossRef] 
31. Giménez, M.J.; Valverde, J.M.; Valero, D.; Zapata, P.J.; Castillo, S.; Serrano, M. Postharvest methyl salicylate treatments delay ripening and maintain quality attributes and antioxidant compounds of 'Early Lory' sweet cherry. Postharvest Biol. Technol. 2016, 117, 102-109. [CrossRef]

32. Yang, Y.-X.; Ahammed, G.J.; Wu, C.; Fan, S.; Zhou, Y.-H. Crosstalk among Jasmonate, Salicylate and Ethylene Signaling Pathways in Plant Disease and Immune Responses. Curr. Protein Pept. Sci. 2015, 16, 450-461. [CrossRef] [PubMed]

33. Rudell, D.R.; Mattheis, J.P.; Fan, X.; Fellman, J.K. Methyl Jasmonate Enhances Anthocyanin Accumulation and Modifies Production of Phenolics and Pigments InFuji'Apples. J. Am. Soc. Hort. Sci. 2002, 127, 435-441. [CrossRef]

34. Ni, J.; Zhao, Y.; Tao, R.; Yin, L.; Gao, L.; Strid, A.; Qian, M.; Li, J.; Li, Y.; Shen, J. Ethylene mediates the branching of the jasmonate-induced flavonoid biosynthesis pathway by suppressing anthocyanin biosynthesis in red Chinese pear fruits. Plant Biotechnol. J. 2020, 18, 1223-1240. [CrossRef] [PubMed]

35. Flores, G.; del Castillo, M.L.R. Influence of preharvest and postharvest methyl jasmonate treatments on flavonoid content and metabolomic enzymes in red raspberry. Postharvest Biol. Technol. 2014, 97, 77-82. [CrossRef]

36. Wang, S.Y.; Bowman, L.; Ding, M. Methyl jasmonate enhances antioxidant activity and flavonoid content in blackberries (Rubus sp.) and promotes antiproliferation of human cancer cells. Food Chem. 2008, 107, 1261-1269. [CrossRef]

37. Yuan, H.; Zhang, J.; Nageswaran, D.; Li, L. Carotenoid metabolism and regulation in horticultural crops. Hortic. Res. 2015, 2, 1-11. [CrossRef]

38. Luo, H.; He, W.; Li, D.; Bao, Y.; Riaz, A.; Xiao, Y.; Song, J.; Liu, C. Effect of methyl jasmonate on carotenoids biosynthesis in germinated maize kernels. Food Chem. 2020, 307, 125525. [CrossRef]

39. Chen, C.; Chen, Z. Potentiation of Developmentally Regulated Plant Defense Response by AtWRKY18, a Pathogen-Induced Arabidopsis Transcription Factor. Physiol. Plant 2002, 129, 706-716. [CrossRef]

40. Du, L.; Chen, Z. Identification of genes encoding receptor-like protein kinases as possible targets of pathogen-and salicylic acid-induced WRKY DNA-binding proteins in Arabidopsis. Plant J. 2000, 24, 837-847. [CrossRef]

41. Robatzek, S.; Somssich, I.E. A new member of the Arabidopsis WRKY transcription factor family, AtWRKY6, is associated with both senescence-and defence-related processes. Plant J. 2001, 28, 123-133. [CrossRef]

42. Xie, T.; Chen, C.; Li, C.; Liu, J.; Liu, C.; He, Y. Genome-wide investigation of WRKY gene family in pineapple: Evolution and expression profiles during development and stress. BMC Genom. 2018, 19, 490. [CrossRef] [PubMed]

43. Yu, D.; Chen, C.; Chen, Z. Evidence for an Important Role of WRKY DNA Binding Proteins in the Regulation of NPR1 Gene Expression. Plant Cell 2001, 13, 1527-1540. [CrossRef] [PubMed]

44. Li, J.; Brader, G.; Palva, E.T. The WRKY70 Transcription Factor: A Node of Convergence for Jasmonate-Mediated and Salicylate-Mediated Signals in Plant Defense. Plant Cell 2004, 16, 319-331. [CrossRef] [PubMed]

Publisher's Note: MDPI stays neutral with regard to jurisdictional claims in published maps and institutional affiliations.

(C) 2020 by the authors. Licensee MDPI, Basel, Switzerland. This article is an open access article distributed under the terms and conditions of the Creative Commons Attribution (CC BY) license (http://creativecommons.org/licenses/by/4.0/). 